\title{
RELAÇÃO ENTRE FAMÍLIA, ESCOLA, ESPECIALISTAS E 0 DESENVOLVIMENTO DE PESSOAS AUTISTAS
}

\author{
Gisele Soares Lemos Shaw iD 1
}

\section{Resumo}

Avanços em estudos sobre Transtorno do Espectro Autista (TEA) indicam contribuições e desafios de familiares, de educadores e de especialistas para auxiliar no desenvolvimento de pessoas autistas. Buscou-se, por meio de revisão integrativa da literatura, investigar as possíveis relações entre família, escola e especialistas no desenvolvimento dessas pessoas. A busca foi realizada nos sites EBSCO Discovery Service (EDS), Pubmed, Google Scholar e CAPES Periódicos, a partir dos descritores "autismo" + "família" + "escola" + "especialista" + "revisão" + "desenvolvimento", escritos em português e em inglês, consideradas publicações feitas entre os anos de 2016 e 2019. Foram selecionados e analisados 20 trabalhos em português e em inglês versando sobre a temática. Os dados foram tratados pelo método de análise de conteúdo. Indicou-se que por meio da aquisição de conhecimentos, da colaboração mútua e do enfrentamento de desafios da condição pelos mencionados grupos, é possível auxiliar no desenvolvimento de pessoas autistas.

Palavras-chave: Autismo; Revisão; Transtorno.

\section{RELATIONSHIP BETWEEN FAMILY, SCHOOL, SPECIALISTS AND THE DEVELOPMENT OF AUTISTIC PEOPLE}

\section{Abstract}

Advances in studies on Autistic Spectrum Disorder (ASD) indicate contributions and challenges from family members, educators and specialists to assist in the development of autistic people. Through an integrative literature review, we sought to investigate the possible relationships between family, school and specialists in the development of autistic people. The search was carried out on the websites EBSCO Discovery Service (EDS), Pubmed, Google Scholar and CAPES Periodicals using the descriptors "autism" + "family" + "school" + "specialist" + "review" + "development", written in Portuguese and in English, considered as a time frame the publications made between the years 2016 and 2019. Twenty articles in Portuguese and English were selected and analyzed dealing with the theme. The data was treated using the content analysis method. It was indicated that through the acquisition of knowledge, mutual collaboration and facing challenges of the condition by the aforementioned groups, it is possible to assist in the development of autistic people.

Keywords: Autism; Disorder; Review.

${ }^{1}$ Doutorado em Educação em Ciências: química da vida e saúde, Universidade Federal do Rio Grande do Sul (UFRGS), Professora Adjunta da Universidade Federal do Vale do São Francisco (UNIVASF), Colegiado de Ciências da Natureza, Senhor do Bonfim, Bahia. E-mail: gisele.shaw@univasf.edu.br.

(9) (1) Perspectivas em Diálogo, Naviraí, v. 8, n. 16, p. 183-201, jan./abr. 2021. 


\section{Introdução}

Avanços em estudos sobre o Transtorno do Espectro do Autismo (TEA) demonstraram contribuições de familiares, de escolas e de especialistas no desenvolvimento de pessoas autistas (ALVERSON; LINDSTROM; HIRANO, 2015, GRASU, 2018, HEYS et al., 2016, OOI et al., 2016, RORIZ, 2016, SOARES, 2016). Os desafios do transtorno são diversos, dada a multifatorialidade e a heterogeneidade do mesmo. Esses enfrentamentos perpassam desde o diagnóstico, a aceitação, o luto, o tratamento de sintomas do TEA, a educação, o desenvolvimento dos indivíduos e sua inclusão social (ALVERSON; LINDSTROM; HIRANO, 2015, LOPES, 2018, MATIAS; PROBST, 2018, OOI et al., 2016).

Assim, tendo em vista analisar um arcabouço de pesquisas que possam basilar investigação empírica sobre as relações entre família, escola e especialistas no desenvolvimento de pessoas autistas, foi realizada revisão integrativa da literatura, com base em procura efetivada em sites de busca da internet. A pesquisa teve como objetivo principal investigar possíveis contribuições de familiares, educadores e terapeutas no desenvolvimento de indivíduos com TEA.

Os dados coletados foram analisados pelo método de análise de conteúdo de Bardin (1977), a partir de categorias de análise definidas a posteriori. A seguir, são apontados caminhos da pesquisa e, posteriormente, indicados resultados, análises e considerações do estudo.

\section{Metodologia}

Este artigo constitui revisão integrativa da literatura, que propicia a busca organizada, síntese e crítica acerca de um tema. Esse método de pesquisa possibilita que um tema seja explorado e refletido, de modo a sintetizar pesquisas disponíveis sobre ele e possibilitar a aplicação prática desses conhecimentos (MENDES; SILVEIRA; GALVÃO, 2008, SOUZA; SILVA; CARVALHO, 2010).

A revisão compõe parte de uma pesquisa maior, de natureza qualitativa, realizada no interior da Bahia, que procura entender as relações família, escola e especialistas no desenvolvimento de pessoas autistas1. Considera-se, nesse estudo, a definição de TEA conforme DSM-5, como um transtorno do desenvolvimento neurológico abrangido por um espectro de quadros, marcados por déficits em dois domínios: deficiências sociais e de comunicação, e interesses restritos, fixos e intensos e comportamentos repetitivos (APA, 2014).

Como exposto anteriormente, buscou-se explorar resposta ao seguinte problema: Quais as possíveis relações entre família, escola e especialistas no desenvolvimento de pessoas autistas tratadas na literatura entre os anos de 2016 e 2019? Para isso, a busca partiu dos descritores "autismo" +"família" +"escola"+ "especialista"+ "revisão"+ "desenvolvimento", escritos em português e em inglês, delimitados trabalhos entre os anos de 2016 e 2019. Foram pesquisados os seguintes sites de busca: EBSCO Discovery Service (EDS), Pubmed, Google Scholar e CAPES Periódicos.

1 O projeto de pesquisa Família, escola e especialistas: relações e possibilidades no desenvolvimento da pessoa autista teve aprovação do Comitê de Ética em Pesquisa da Fundação Universidade Federal do Vale do São Francisco (UNIVASF), sob o registro CAAE: 00943418.7.0000.5196.

(c) (1)(3) Perspectivas em Diálogo, Naviraí, v. 8, n. 16, p. 183-201, jan./abr. 2021. 
Como critérios de inclusão dos trabalhos, foram selecionados artigos e/ou trabalhos de conclusão de curso (TCC) que forneciam informações sobre as relações entre, pelo menos, um dos três grupos - família, escola ou especialistas no desenvolvimento de pessoas com TEA. Já como critério de exclusão, não foram elegidas obras de difícil acesso, aquelas que não fossem possíveis ser baixadas gratuitamente da internet ou que os autores, após contatados, não as disponibilizassem. Os TCCs foram considerados, tendo em vista que foram avaliados por banca de professores especialistas, assim como os artigos e suas discussões, que se mostraram essenciais na análise.

Quando pesquisadas as palavras-chave em português, no EBSCO Discovery Service (EDS) foram encontrados oito resultados. Analisando os títulos e resumos, foram excluídos três artigos que somente mencionaram sobre autismo. Na busca ao PUBMED não houve resultados. Pesquisas realizadas no site Google scholar levaram à obtenção de 975 trabalhos. Foram escolhidos os 50 primeiros trabalhos exibidos (considerando o filtro de relevância do Google), dentre os quais, um livro foi excluído, pela dificuldade de acesso. Somente foram selecionados 50 trabalhos, dentre os 975, para possibilitar análise mais cuidadosa, visto que o objetivo da revisão foi a análise qualitativa, e não a avaliação quantitativa das obras. Em meio aos 49 trabalhos restantes, 14 foram elencados para ser lidos integralmente, com base na análise de título e resumo. Já no site CAPES periódicos foram achados quatro trabalhos que não tratavam do assunto. Assim, foi obtido um total de 17 produções escritas em português para leitura, sendo que uma delas foi excluída por sua abordagem superficial do assunto. Os dez primeiros trabalhos resultantes foram selecionados para revisão, levando em consideração que a análise de uma quantidade superior de trabalhos dificultaria a publicação em periódico científico. Foi levado em consideração que a qualidade da discussão do produto final, o artigo científico, seria mais importante que a quantidade de pesquisas mencionadas. Desse modo, foram excluídos os trabalhos que suplantaram os dez primeiros insurgentes na pesquisa.

Depois, foi realizada busca nos mesmos portais mencionados com os descritores "autism" + "family" + "school" + "specialist" + "review. Na base de periódicos da Capes foram obtidos 941 resultados e, dos 30 primeiros trabalhos, 17 foram separados pela leitura de títulos e abstracts. A seleção dos trinta primeiros trabalhos se deu pelo critério de relevância do Google, considerando o objetivo de realizar seleção qualitativa e não quantitativa de obras. Após leitura na íntegra, foram excluídos três deles, por não tratarem especificamente da questão de estudo. Foram elencados os dez primeiros trabalhos em inglês para a revisão, em decorrência da importância de delimitar quantidade de publicações que possibilitasse avaliação cuidadosa e publicação do artigo resultante em periódico científico, que não permite publicações muito extensas.

Assim, após minucioso trabalho de triagem, foram selecionadas 20 obras para compor a revisão. Após leitura das mesmas, elas foram fichadas e foram selecionadas as seguintes informações: relações trazidas entre os elementos família, escola e especialistas, materiais e métodos utilizados, resultados encontrados e considerações dos autores. Depois, após novas leituras, buscou-se responder à questão de pesquisa a partir do conteúdo de cada trabalho. Desse exame, foram destacadas categorias, que foram descritas e divididas em subcategorias. Esse conteúdo foi organizado e interpretado. 


\section{Contribuições da família, da escola e de especialistas no desenvolvimento da pessoa autista: resultados e discussões}

Após minuciosa seleção das obras que poderiam responder à questão de pesquisa foram analisados os seguintes trabalhos, elencados no quadro 1.

Quadro 1: Trabalhos incluídos na revisão.

\begin{tabular}{|c|c|c|c|}
\hline Referência & Tipo de trabalho & $\begin{array}{l}\text { Relações } \\
\text { envolvidas }\end{array}$ & Métodos \\
\hline $\begin{array}{l}\text { Alverson, Lindstrom e } \\
\quad \text { Hirano (2015) }\end{array}$ & Artigo & $\begin{array}{l}\text { Família, escola, } \\
\text { especialistas }\end{array}$ & $\begin{array}{c}\text { Pesquisa qualitativa, entrevistas, } \\
\text { questionários e registros }\end{array}$ \\
\hline Casanova (2016) & $\begin{array}{l}\text { Relatório de } \\
\text { mestrado }\end{array}$ & Família, escola & $\begin{array}{l}\text { Estudo de caso, estudo teórico e } \\
\text { análise de documentos. }\end{array}$ \\
\hline $\begin{array}{c}\text { Clark, Barbaro e } \\
\text { Dissanayake (2017) }\end{array}$ & Artigo & Especialistas & $\begin{array}{l}\text { Pesquisa quantitativa, com } \\
\text { investigação longitudinal e uso de } \\
\text { escalas }\end{array}$ \\
\hline Cruz (2015) & $\begin{array}{l}\text { Monografia de } \\
\text { especialização }\end{array}$ & Família, escola & $\begin{array}{c}\text { Estudo de caso, uso de entrevistas } \\
\text { e questionário sobre o cotidiano } \\
\text { da criança. }\end{array}$ \\
\hline $\begin{array}{l}\text { Dillon, Underwood e } \\
\text { Freemantle (2014) }\end{array}$ & Artigo & Família, escola & $\begin{array}{c}\text { Pesquisa mista, questionários e } \\
\text { entrevistas }\end{array}$ \\
\hline Grasu (2018) & Artigo & $\begin{array}{l}\text { Família, escola, } \\
\text { especialistas }\end{array}$ & Pesquisa qualitativa, entrevistas \\
\hline Heys et al. (2016) & Artigo & $\begin{array}{l}\text { Família, escola, } \\
\text { especialistas }\end{array}$ & $\begin{array}{l}\text { Pesquisa qualitativa, entrevistas } \\
\text { semiestruturadas em grupo focal }\end{array}$ \\
\hline Lopes (2018) & Artigo & $\begin{array}{l}\text { Família, } \\
\text { especialistas }\end{array}$ & Pesquisa bibliográfica \\
\hline $\begin{array}{c}\text { Matias e Probst } \\
(2018)\end{array}$ & Artigo & Família, escola & $\begin{array}{l}\text { Pesquisa qualitativa, de revisão } \\
\text { bibliográfica }\end{array}$ \\
\hline $\begin{array}{l}\text { Mousinho, Adous e } \\
\text { Gikovate (2016) }\end{array}$ & Artigo & Família, escola & Estudo de caso \\
\hline Nascimento (2017) & $\begin{array}{l}\text { Monografia de } \\
\text { graduação em } \\
\text { Pedagogia }\end{array}$ & Família, escola & Estudo de caso \\
\hline Ooi et al. (2016) & Artigo & $\begin{array}{l}\text { Família, escola, } \\
\text { especialistas }\end{array}$ & Revisão bibliográfica \\
\hline $\begin{array}{c}\text { Perera, Jeewandara, } \\
\text { Senevirat e Guruge } \\
\text { (2016) }\end{array}$ & Artigo & $\begin{array}{l}\text { Família, } \\
\text { especialistas }\end{array}$ & $\begin{array}{l}\text { Pesquisa quantitativa com } \\
\text { intervenção clínica }\end{array}$ \\
\hline Ravet (2017) & Artigo & Escola & $\begin{array}{c}\text { Pesquisa qualitativa com aplicação } \\
\text { de questionários e entrevistas em } \\
\text { grupos focais }\end{array}$ \\
\hline Ribeiro (2018) & $\begin{array}{l}\text { Monografia de } \\
\text { graduação em } \\
\text { Pedagogia }\end{array}$ & Família, escola & Revisão bibliográfica \\
\hline Roriz (2016) & $\begin{array}{l}\text { Dissertação de } \\
\text { mestrado }\end{array}$ & $\begin{array}{l}\text { Família, escola e } \\
\text { especialistas }\end{array}$ & Revisão bibliográfica \\
\hline $\begin{array}{l}\text { Santos, Leão e } \\
\text { Agapito (2018) }\end{array}$ & Artigo & Família, escola & $\begin{array}{c}\text { Pesquisa qualitativa, entrevistas } \\
\text { semiestruturadas }\end{array}$ \\
\hline
\end{tabular}




\begin{tabular}{|c|c|c|c|}
\hline Soares (2016) & $\begin{array}{l}\text { Monografia de } \\
\text { graduação em } \\
\text { Psicologia }\end{array}$ & $\begin{array}{l}\text { Família, escola, } \\
\text { especialista }\end{array}$ & Pesquisa histórica \\
\hline $\begin{array}{c}\text { Townsend e } \\
\text { Puymbroeck (2017) }\end{array}$ & Artigo & Família & $\begin{array}{c}\text { Pesquisa qualitativa com } \\
\text { entrevistas e análise de anotações } \\
\text { de observação }\end{array}$ \\
\hline Shochet et al. (2016) & Artigo & Família, escola & Revisão bibliográfica \\
\hline
\end{tabular}

Fonte: Arquivo da autora (2020).

A maioria deles constituiu-se de artigos publicados em periódicos, entre 2016 e 2019. Os demais trabalhos foram três monografias de cursos de graduação, dois deles em Pedagogia e um deles em Psicologia, uma monografia de curso de especialização e dois trabalhos de conclusão de curso de mestrado (CASANOVA, 2016, RORIZ, 2016).

Acerca dos grupos e relações envolvidas nos trabalhos, apenas seis deles trouxeram elementos que tratam das contribuições de família, escola e especialistas; nove deles abordaram sobre família e escola; e os outros exploraram questões relativas a apenas um dos grupos. A grande maioria dos trabalhos foi proveniente de pesquisas qualitativas $(n=17)$, sendo duas pesquisas quantitativas e uma mista. Dos trabalhos qualitativos analisados, cinco deles constituíram, especificamente, revisão bibliográfica e um deles compôs pesquisa histórica (conforme quadro 1). 0 quadro 2 ilustra o teor de cada um dos trabalhos selecionados.

Quadro 2: Teor das pesquisas que originaram os trabalhos analisados.

\section{Autores}

\begin{tabular}{c} 
Alverson, \\
Lindstrom e \\
Hirano (2015) \\
\hline $\begin{array}{c}\text { Casanova } \\
\text { (2016) }\end{array}$
\end{tabular}

Clark, Barbaro e
Dissanayake
(2017)

Cruz (2015)

Dillon,

Underwood e

Freemantle

(2014)

Grasu (2018)
Investigaram experiências de transição do ensino médio para o ensino superior de cinco jovens, com idades entre 19 a 22, diagnosticados com Síndrome de Asperger (SA), por meio de questionário com familiares, revisão de registros de educação especial e entrevistas individuais $(N=27)$ com jovens adultos com SA, membros da família, professores e conselheiros de reabilitação dos mesmos.

Analisou caso de criança autista, com base no qual escreveu seu relatório de conclusão de curso de mestrado em ciências da educação, especializada em educação especial.

O estudo investigou aspectos cognitivos e comportamentais de 48 crianças diagnosticadas com TEA, o progresso do desenvolvimento da gravidade dos sintomas de cognição e autismo a partir de 24 meses da idade escolar e a estabilidade dos sintomas do TEA longitudinalmente.

Buscou entender o quanto as relações escola-família podem auxiliar no processo de inclusão de crianças com deficiência intelectual. Investigou caso de uma criança de seis anos com TEA, entrevistando professora da sala regular, professora da sala de Atendimento Educacional Especializado (AEE) e a mãe da criança.

Pesquisaram experiências dominantes de 14 pessoas diagnosticadas com TEA no ensino secundário em comparação a experiências de 14 pessoas neurotípicas, utilizando auto relato, por meio de questionários e entrevistas semiestruturadas.

Realizou estudo a partir do acompanhamento psicossocial de crianças com autismo por quase dois anos, em quatro centros de assistência. Foram entrevistados 25 profissionais e pais das crianças autistas. 


\begin{tabular}{c}
$\begin{array}{c}\text { Heys et al. } \\
\text { (2016) }\end{array}$ \\
\hline Lopes (2018) \\
\hline $\begin{array}{c}\text { Matias e Probst } \\
\text { (2018) }\end{array}$ \\
Mousinho, \\
Adous e \\
Gikovate (2016) \\
Nascimento \\
(2017) \\
Ooi et al. \\
(2016) \\
Perera, \\
Jeewandara, \\
Senevirat e \\
Guruge (2016)
\end{tabular}

\begin{tabular}{|l|}
\hline Ravet (2017) \\
\hline Ribeiro (2018) \\
\hline
\end{tabular}

Roriz (2016)

Santos, Leão e Agapito (2018)

Soares (2016)

Townsend e Puymbroeck (2017)

Shochet et al. (2016)

Investigaram a compreensão de pais e profissionais sobre autismo num país de baixa renda, o Nepal. Eles realizaram entrevistas com pais de crianças autistas e não autistas e profissionais da educação e saúde $(n=106)$, questionando-os acerca de desenvolvimento típico e atípico.

Discutiu a importância da família para o desenvolvimento da autonomia da pessoa com Síndrome de asperger.

Apresentaram a heterogeneidade das pessoas com TEA apresentando processos educativos, particularidade, fatos históricos, sintomas e características.

Investigou estudante de faculdade de música, autista e cego, descrevendo o percurso de ensino e aprendizagem e seus desafios.

Analisou processo de inclusão de uma criança com TEA matriculada no ensino regular em escola particular em João Pessoa. Realizou-se entrevista com a mãe da criança, questionário com cinco professores e observação da criança entre os anos de 2012 e 2017.

Realizou meta-síntese de estudos sobre percepções e experiências de pais de crianças com TEA, evidenciando desafios enfrentados.

Apresentaram um programa de intervenção no autismo, com base no Modelo Denver de Intervenção Precoce (ESDM), desenvolvido no Sri Lanka, em casa, com crianças entre 18 e 40 meses de idade. Os resultados do programa foram comparados com um grupo controle. As mães fizeram um treinamento com três médicos juniores que acompanhamento o processo.

Investigou se um curso de formação inicial de professores do Reino Unido está preparando-os para ensinar pessoas autistas. Trouxe percepções iniciais de estudantes e professores do curso sobre a educação para autismo, por meio de questionário e entrevista em grupo focal.

Investigou a inclusão escolar de pessoas autistas por meio de artigos na base de dados Scientific Eletronic Library Online (SCIELO Brasil).

Analisou artigos entre 2000 e 2016, principalmente do PubMed, em sites de associação de autistas, entre outros evidenciando etiologia, sintomatologia, diagnóstico e tratamento da Perturbação do Espectro do Autismo.

Investigaram como ocorre 0 atendimento a estudantes com transtorno do espectro autista em escolas públicas de Barra do Bugres/MT e as concepções de profissionais da educação sobre os direitos subjetivos dessas pessoas. Foram realizadas entrevistas semiestruturadas com profissionais de escolas estaduais de ensino comum, APAE e Assessoria Pedagogia do município de Barra do Bugres/MT.

Realizou apanhado histórico da concepção do deficiente, e suas implicações socioculturais, políticas e sociais, tendo como recorte histórico desde a descoberta do TEA até a última revisão do Manual Diagnóstico e Estatístico e Transtornos Mentais (DSM-V).

Por meio de entrevistas com 24 pais e notas do investigador, pesquisaram contribuições do lazer em família para a melhoria do funcionamento familiar de lares que possuem pessoas com TEA, investigando percepções de pais de crianças autistas que participaram de experiência de lazer num acampamento, com duração de dois dias e noites.

Trouxeram inúmeras pesquisas que tratam da promoção da Saúde Mental para adolescentes com TEA.

Fonte: Arquivo da autora (2020).

Após análise de conteúdos contidos nas obras, sugiram três categorias de análise: conhecimento, colaboração e desafios. A subcategoria colaboração e a 
subcategoria desafios foram apresentadas por meio da divisão por temas - família, escola e especialistas, para facilitar o entendimento (conforme quadro 3 ).

\section{Quadro 3: Categorias elencadas na revisão após análise de conteúdo.}

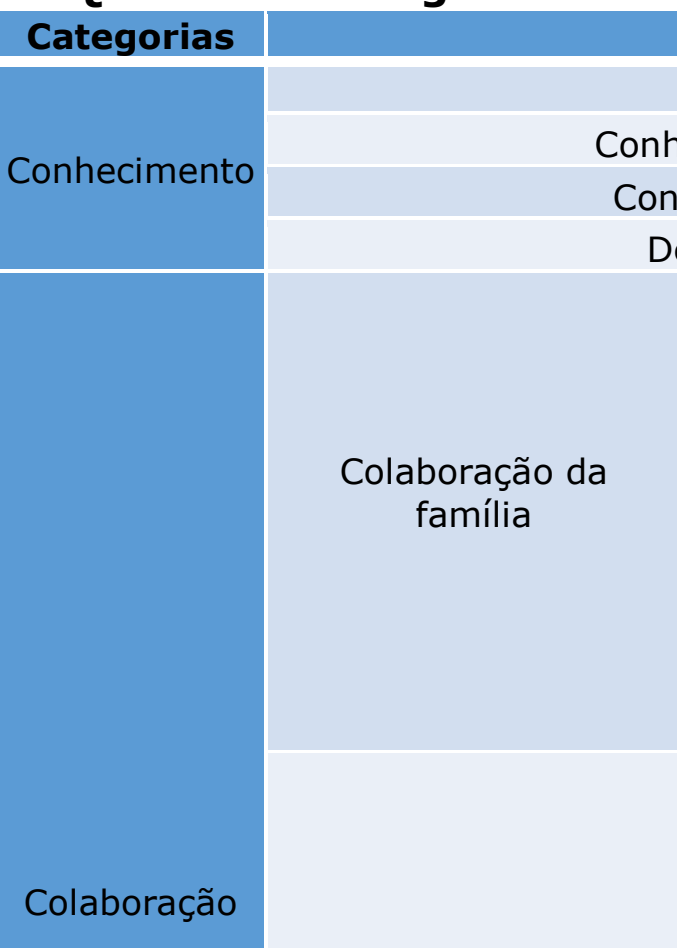

Colaboração da escola

Colaboração dos especialistas

Desafios da família

Desafios

\section{Subcategorias}

Conhecer sobre TEA

Conhecer características de pessoas com TEA

Conhecer como lidar com pessoas com TEA

Desenvolver autoconsciência sobre TEA

Cuidar do indivíduo

Incentivar

Promover desenvolvimento da autonomia

Realizar mediação social

Gerenciar o desenvolvimento físico e psíquico do indivíduo

Administrar tratamento

Complementar tratamento no âmbito doméstico

Dialogar com escola e especialistas

Permitir visão integral do indivíduo

Alicerçar desenvolvimento cognitivo

Promover vínculos sociais

Ênfase nas habilidades

Contornar desafios

Incentivar

Desenvolver habilidades sociocognitivas

Permitir visão integral do indivíduo

Combater bullying

Dialogar com a família

Realizar mediação social

Adaptar programas

Realizar diagnóstico

Auxiliar na superação da negação de pais

Realizar tratamento no indivíduo

Propiciar visão integral do indivíduo

Facilitar vínculos entre pais, crianças e escola

Aceitar o diagnóstico

Lidar com sintomas do TEA

Buscar especialistas

Aceitar a desordem

Lidar com negação, culpa, depressão e estresse

Gerir problemas de comportamento do indivíduo com TEA

Desenvolver estratégias de enfrentamento (coping)

Lidar com o preconceito

Promover formação em TEA

Realizar inclusão e educação especial

Desafios da escola

Fomentar apoio administrativo e de professores

Gerir bem o tempo escolar

Lidar com sintomas do TEA 
Conseguir apoio do Estado

Desafios dos especialistas

Fonte: Arquivo da autora (2019).
Identificar comorbidades associadas

\subsection{Conhecimento}

A categoria conhecimento tratou da importância da aquisição desse elemento para família, escola e especialistas. Ele foi mencionado como imprescindível para auxiliar no desenvolvimento da pessoa autista, seja no entendimento do que seja TEA e suas características em pessoas autistas, como em lidar com elas, no processo de conscientização social e autoconsciência do autismo. Para Heys et al. (2016), é preciso que a sociedade seja capacitada acerca do autismo. Sua pesquisa apontou as necessidades de capacitação e de aumento de financiamento para realização de terapias para melhorar a qualidade de vida de pessoas com TEA. Todos os profissionais entrevistados admitiram não ter conhecimento suficiente sobre o transtorno. Também, Ooi et al. (2016) observaram que pais enfrentaram estigmatização social de pessoas que os julgavam como maus pais, devido a comportamentos disruptivos de seus filhos. Muitos daqueles mencionaram que a falta de compreensão sobre autismo ocasiona esses estereótipos e outros acreditam que isso se dá pelo fato de o autismo não ser visível por meio da aparência das crianças.

A falta de conhecimento e de compreensão sobre autismo foram mencionados como os maiores causadores da falta de eficiência das escolas em lidar com a inclusão escolar, e o despreparo de professores está presente, inclusive, em cursos de formação inicial (RAVET, 2017). Alverson, Lindstrom e Hirano (2015), destacaram que a autoconsciência sobre o transtorno pode ajudar o autista a conhecer suas limitações, buscar ajuda e, assim, facilitar sua inclusão social. Ainda, de acordo com Dillon; Underwood e Freemantle (2014), experiências comparativas de escolares com e sem TEA demonstraram que a conscientização da comunidade escolar sobre autismo pode melhorar a experiência escolar e o sucesso de pessoas com o transtorno.

Além de conhecer sobre o transtorno, é preciso que as pessoas entendam as características apresentadas por pessoas autistas. Ravet (2017) esclareceu que pessoas com TEA possuem modos diferenciados de experienciar o mundo e, por isso, é preciso que os professores as conheçam e as entendam, fazendo adequações necessárias em seu ensino e no currículo. Ele esclareceu que, sem essas adaptações, é difícil que autistas consigam conviver em classes barulhentas e sem sentido para elas, por serem locais que geram estresse e ansiedade nos mesmos (RAVET, 2017). Essa inadaptação escolar faz com que muitas vezes os pais não matriculem seus filhos em escolas regulares e, alguns deles, até achem que seus filhos podem perturbar a aprendizagem de crianças típicas (HEYS et al, 2016).

Também é necessário que familiares e profissionais da saúde e da educação saibam como lidar com pessoas autistas dentro de seu contexto de trabalho e de suas especialidades, que realizem as adequações e intervenções necessárias e que auxiliem no desenvolvimento dessas pessoas. No caso de familiares, Lopes (2018) explicou a importância de que eles estejam abertos à aprendizagem, respeitando limites e expectativas dos adolescentes, dando-Ihes autonomia e capacidade de 
fazerem escolhas. Dado que cada autista possui características próprias, é preciso que a família desenvolva flexibilidade para aprender com essas especificidades.

Shochet et al. (2016) relataram a existência de três linhas de frente em programa de saúde mental para jovens: aquela com intervenções com o jovem (RAPA1), aquela com ações com a família (RAP-P) e a que intervém com educadores (RAPT). Nesse programa são incentivados sentimentos de pertença, de auto-regulação e de resiliência. Na adaptação da proposta, voltada para jovens com TEA, o conhecimento do transtorno e de suas especificidades é fundamental no processo (SHOCHET, et al., 2016)2.

\subsection{Colaboração}

A categoria colaboração surgiu a partir de mencionadas colaborações de familiares, de escola e de especialistas. Sobre o papel da família nessa colaboração foram destacados nove itens: cuidar do indivíduo, incentivar, promover o desenvolvimento da autonomia, realizar mediação escolar, gerenciar o desenvolvimento físico e psíquico do indivíduo, administrar terapias, complementar essas terapias no âmbito doméstico, dialogar com a escola e com especialistas e permitir visão integral do indivíduo.

$\mathrm{O}$ ato de cuidar da pessoa autista é especialmente destacado quando se trata de cuidar de pessoa com deficiência, tendo em vista que requer cuidados especiais e de gestão do trabalho familiar. De acordo com Nascimento (2017), o cuidado com a criança autista deve ser compartilhado entre a família e a escola e deve auxiliar na redução de sintomas do TEA e no desenvolvimento da autonomia da criança. No estudo de Alverson, Lindstrom e Hirano (2015), as famílias que incentivaram a autonomia de seus filhos foram as que os mantiveram em escolas regulares no percurso escolar, e as que mais ajudaram em seu processo de adaptação ao ensino superior. Também, no caso do garoto autista Pedro, investigado por Cruz (2015), observou-se que o desenvolvimento de sua autonomia promoveu mais liberdade para o mesmo manifestar o que deseja e o que pensa. A autora explicou que a forte participação da mãe de Pedro em seu desenvolvimento, junto ao apoio da escola, foi fundamental. Segundo Lopes (2018): "É importante que a família observe o adolescente, converse com ele, ou melhor, saiba escutá-lo, incentive-o fazer pequenas tarefas [...] trabalhe sua autoestima, auto respeito e autoconhecimento" (LOPES, 2018, p.61).

É importante que haja envolvimento familiar no processo educativo da pessoa com TEA, inclusive na divisão de tarefas em casa. Por outro lado, também é preciso que a família busque se integrar em redes de apoio, que busque suporte psicológico para todos os membros, devido ao estresse familiar desencadeado pela presença do transtorno no grupo (NASCIMENTO, 2017, RORIZ, 2017). Na pesquisa de Towsend e Puymbroeck (2017), foi aferido que participar de rede de apoio junto a outras famílias de pessoas autistas reduziu sentimentos de cobrança e aumentou sentimentos de

1 RAP - Resourceful Adolescent Program. Esse programa envolve o RAP-A para adolescentes (Resourceful Adolescent Program for Adolescents) o RAP-P (Resourceful Adolescent Program for Parents) e o RAP-T (the Resourceful Adolescent Program for Teachers).

${ }^{2}$ A adaptação desse programa para pessoas com TEA é o RAP para jovens com ASD (Autism Spectrum Disorder), que pode ser o RAP-A-ASD, o RAP-P-ASD ou o RAP-T-ASD.

@()@ Perspectivas em Diálogo, Naviraí, v. 8, n. 16, p. 183-201, jan./abr. 2021. 
apoio e de aceitação, já que propiciou o compartilhamento de experiências e de problemas em comum. Segundo Roriz (2016), "A família é o elemento principal de desenvolvimento físico e psicológico para qualquer elemento que esteja nele integrado" ( $p .58$ ). É comum que familiares de pessoas com TEA se deparem com situações que geram estresse e ansiedade, podendo desenvolver depressão, inclusive o que, por vezes, ocorre com irmãos de autistas, que frequentemente não recebem a devida atenção. Assim, além de dividir o trabalho do cuidar e do educar com a escola, a família deve gerenciar terapias com a criança e o tratamento psicológico de seus membros. O processo terapêutico com a pessoa autista é, por si só, um dos grandes desafios, pois abrange desde a busca pelos especialistas até a manutenção do acompanhamento terapêutico e até sua complementação, no âmbito doméstico (NASCIMENTO, 2017, RORIZ, 2017).

A escola é um grupo cuja colaboração é importante para o desenvolvimento da pessoa com TEA. Soares (2016) destacou a função dessa instituição em alicerçar o desenvolvimento cognitivo das pessoas. Porém, muitas vezes, as escolas apenas aceitam a matrícula de pessoas com deficiência, mas sem propiciar-lhes aprendizagens de conteúdos e de habilidades curriculares, tomando para si apenas o papel de ambiente de socialização. É preciso que a escola, apoiada pela família e, inclusive, por especialistas, inclua a criança e/ou o jovem, promovendo seu desenvolvimento cognitivo a partir do estímulo à aprendizagem de conhecimentos e de habilidades, além de auxiliá-los na formação de vínculos positivos, através de relacionamentos que gerem confiança e bem-estar para a pessoa autista, desenvolvidos a partir da compreensão e do acolhimento do indivíduo com TEA (MATIAS; PROBST, 2018, SOARES, 2016). Essa formação de vínculos entre a pessoa com TEA e demais sujeitos da comunidade escolar faz-se essencial, dado que a escola atua como mediadora social do indivíduo.

Alverson, Lindstrom e Hirano (2015) observaram, em casos que estudaram, como a família e a escola foram fundamentais nos processos de transição de jovens autistas do ensino médio para o ensino superior e para vida profissional. No estudo de Cruz (2015), o bom desenvolvimento de Pedro, inclusive na socialização com colegas e professores, foi atribuído à presença da família em conexão com a escola. De acordo com Shochet et al. (2016), pesquisas apontaram que o sentimento de pertença a um grupo social, como a escola, pode proteger o indivíduo de diversos problemas, tais como psicopatia, depressão, estresse, além de auxiliar no desenvolvimento de autoestima, de visão positiva sobre a vida, de expectativas de futuro, de esperança e de melhorar o desempenho acadêmico. Já o sentimento de não pertencimento pode levar à depressão, uso de substâncias ilícitas e suicídio (SHOCHET, et al., 2016).

Devido às peculiaridades das pessoas com TEA, para que a inclusão ocorra nas escolas é preciso que a instituição venha a: adaptar programas conforme características do sujeito (RORIZ, 2016, SOARES, 2016); focar em suas habilidades e não nas dificuldades e limitações do mesmo (NASCIMENTO, 2017); contornar desafios que aparecerem, evitando a mera biologização do transtorno e realizando adaptações (RIBEIRO, 2018); incentivar o sujeito autista a superar dificuldades em sala de aula (DILLON; UNDERWOOD; FREEMANTLE, 2014); e desenvolver habilidades sociocognitivas, a partir do estabelecimento de vínculos e socialização com colegas e professores (NASCIMENTO, 2017). 
De acordo com Soares (2016), algumas manifestações comportamentais de pessoas autistas nas escolas podem causar estranhamento a professores que chegam a duvidar da capacidade de os autistas aprenderem. Muitos desses docentes vêem a escola apenas como local de socialização para os estudantes com TEA. A autora explicou, ainda, que faltam programas que orientem esses professores a fazerem as necessárias adaptações pedagógicas, com base em dificuldades mais presentes, tais como déficit de atenção, dificuldades no raciocínio e problemas na aceitação de erros.

Segundo Casanova (2016), o professor precisa observar "a organização e condições de estimulação do ambiente, as instruções e sinais que dá à criança, as ajudas que proporciona e as motivações e reforços que utiliza para promover a aprendizagem" (p.45). Nesse sentido, a escola precisa estar em consonância com o trabalho dos especialistas e em diálogo com a família, de modo a prover as adequações que a criança com TEA necessitar. Assim, seu papel suplanta o conhecimento sobre o transtorno, que é condição mínima para o processo de inclusão, sendo que sua falta gera outros problemas, tais como o bullying, que precisa ser prevenido e combatido. De acordo com Nascimento (2017), os professores precisam ficar atentos ao bullying, porque muitas vezes as crianças autistas, por conta de dificuldades de entender pensamentos alheios, não entendem intenções de escárnio e de gozação. Para Roriz (2016), a cooperação entre profissionais e o apoio da família são essenciais tanto na orientação do indivíduo com TEA durante toda sua vida, quanto para possibilitar a visão integral do indivíduo, como um ser inteiro, muito maior do que a existência do transtorno.

Além de auxiliar familiares e educadores a promoverem uma visão mais sistêmica do ser autista (RORIZ, 2016), o time de especialistas deve ser responsável por colaborar com diversas questões, desde a realização precisa do diagnóstico, a partir da utilização de métodos de rastreio adequados, em auxiliar os pais a superarem a negação do diagnóstico (RORIZ, 2016) e até na realização das terapias (NASCIMENTO, 2017, PERERA et al., 2016). Para Lopes (2018), apesar de muitos autistas não terem atrasos na linguagem e/ou déficit cognitivo, faz-se importante 0 diagnóstico precoce, para que eles possam atingir seu potencial. Clark, Barbaro e Dissanayake (2017) notaram estabilidade de sintomas e da gravidade do autismo desde a infância até a idade escolar de crianças investigadas, que elas atribuíram à amenização de sintomas do autismo, melhorando a qualidade de vida do indivíduo autista.

Além da intervenção em sintomas do TEA por meio de terapia comportamental, fonoaudiológica e ocupacional, entre outras, às vezes, é preciso controlar sintomas de autismo por meio do uso de fármacos, prescritos por médicos especialistas. De acordo com Roriz (2016), pelas crianças autistas muitas vezes terem quantidades anormais de serotonina e outros neurotransmissores, muitas vezes é necessário ministrar fármacos, geralmente psicoestimulantes, inibidores seletivos da recaptação da serotonina e antipsicóticos para reduzir a incidência de sintomas que dificultem a vida da pessoa.

Além do mais, especialistas podem colaborar no desenvolvimento da pessoa autista a partir da facilitação de vínculos entre pais, crianças e escola. Soares (2016) incentivou o papel do psicanalista na terapia, que pode ser mediador da relação família, escola, analista, num lugar de invenção de soluções (SOARES, 2016). 


\subsection{Desafios}

A terceira categoria elencada na pesquisa, desafios, envolve dificuldades da família, da escola e de especialistas em lidar com o TEA. Para Roriz (2016), deve haver compartilhamento de conhecimento e cooperação entre família, escola e especialistas, tanto no diagnóstico, quanto no tratamento de sintomas do autismo e na inclusão social das pessoas com TEA (RORIZ, 2016).

A família é o grupo que mais deve encarar desafios do transtorno. Tudo se inicia com a aceitação do diagnóstico, que muitas vezes não ocorre, prevalecendo a fase de negação, o que pode inviabilizar as terapias e o desenvolvimento do indivíduo no espectro. Grasu (2018) explicou que os pais passam por processo de negação, culpa e depressão, por saberem que o autismo muitas vezes traz sintomas que geram desordens na vida do indivíduo e, muitas vezes, eles buscam uma segunda opinião médica, atrasando o tratamento desses sintomas. No estudo de Ooi et al. (2016), ao receberem diagnóstico de autismo, os pais ficaram espantados e com raiva, sendo que muitos deles se sentiram culpados ou desesperançados. Soares (2016) mencionou sentimento de frustração, medo e confusão de pais na descoberta do autismo, a necessidade de buscar especialistas e o enfrentamento do preconceito, 0 denominado luto. De acordo com Silva, Gaiato e Reveles (2012), nessa situação, o luto representa a perda de uma criança sem deficiência, idealizada pela família, e o abandono dessa imagem de criança que nunca existiu. Lopes (2018) orientou que, nessa etapa de luto, "as pessoas envolvidas precisam conhecer as características do TEA e aprender técnicas que facilitam (...) o relacionamento entre todos que com ela convivem" (p.59). Após instalarem-se sentimentos de medo, vergonha e culpa e superarem a tristeza pela perda de um filho perfeito fantasiado, ocorre a adaptação e reorganização, que são fundamentais para o tratamento de sintomas que dificultam a qualidade de vida do autista (RORIZ, 2016, p.58).

Aceitando ou não o diagnóstico de TEA, a família tem que lidar com sintomas provenientes do transtorno que podem surgir, tais como: heteroagressão ou autoagressão, agitação, comportamento opositivo, insônia e problemas para se alimentar (GRASU, 2018). Nascimento (2017) verificou diversos sinais de TEA apresentados por criança estudada: dificuldades de interação, agressividade, interesses restritos a desenhos e animais, utilização de pessoas como instrumento, dificuldades de linguagem, movimentos estereotipados e repetitivos, locomoção nas pontas dos pés, organização de objetos em fileiras, dificuldade de olhar para câmera fotográfica, agitação com mudanças de rotina, isolamento, dificuldade em manter olhar fixo, dificuldade em se manter em sala de aula, incômodo com barulhos, risos inadequados, ecolalias, inversão pronominal (NASCIMENTO, 2017). De acordo com Ooi et al. (2016), comportamentos frequentes de crianças com TEA foram classificados por seus pais como difíceis, desafiadores e geradores de estresse no cotidiano da família. Também foram mencionadas birras e agressões incontroláveis, além de episódios de destruição de coisas da casa e violência. Porém, muitos desses comportamentos são desencadeados devido a dificuldades geradas por conta de hipersensibilidade sensorial ou ocasião de mudanças de rotina ou de ambiente não antecipadas às pessoas autistas, problemas esses que podem ser evitados pela adoção de rotinas estruturadas, ajustes domésticos e alertas (OOI et al., 2016).

De acordo com Shochet et al. (2016), as dificuldades de socialização advindas dos sintomas do TEA junto às pressões sociais, essas bastante comuns nessa idade, 
tornam adolescentes autistas mais propícios à solidão e ao isolamento, tornando-os mais vulneráveis à depressão e à ansiedade. Estudos classificaram adolescentes com TEA com níveis mais altos de agressividade, comportamentos desafiadores, dificuldades de atenção, menor desempenho acadêmico e mais sintomas de depressão e ansiedade do que seus colegas neurotípicos (SHOCHET et al., 2016). É preciso destacar que as especificidades de pessoas autistas nem sempre são negativas, como amplamente abordadas na literatura: "(...) uma característica comum é que o indivíduo se torna um especialista no assunto que elegeu e apresenta facilidade para o reconhecimento de padrões de repetição de estímulos" (MOUSINHO; ADOUR; GIKOVATE, 2016, p.197).

No estudo de Ooi et al. (2016), pais de autistas caracterizaram seus filhos como carinhosos, inteligentes e bem-humorados. Eles também afirmaram que se tornaram pessoas mais sensíveis, compreensivas e menos críticas com as pessoas ao redor e também mais pacientes, sociáveis e autoconfiantes. Apesar de pessoas autistas não se resumirem a sintomas do TEA, a sociedade costuma evidenciar caracerísticas do transtorno, talvez pela incompreensão do mesmo.

Mas, para amenização desses sintomas, é preciso que as famílias enfrentem a busca por especialistas. Isso representa encontrar profissionais especializados que realizem o tratamento de sintomas, além de realizar complementações necessárias em ambiente doméstico. Grasu (2018) apontou que a intervenção precoce em crianças com TEA aumentou as possibilidades de integração social de autistas, além de ter propiciado a redução dos níveis de estresse e depressão entre seus familiares, principalmente mães, as principais cuidadoras. De acordo com Grasu (2018), muitas vezes, após o diagnóstico, os pais não sabem onde realizar as terapias com seus filhos e, às vezes, eles encontram locais com serviços, mas que geram custos que não podem ser arcados, ou encontram serviços que abrangem grande número de crianças, o que não é adequado a pessoas autistas.

O estudo de Oii et al. (2016) demonstrou que muitas mães de autistas tiveram que deixar seus empregos, apesar dos gastos recorrentes com tratamentos de sintomas de TEA, cuidados e pagamento de danos. Além do lazer e da saúde dos pais serem afetados pelo excesso de demandas e falta de cuidados consigo, gerando privação de sono, baixa energia e insônia (OOI et al., 2016). Além de lidarem com sintomas, os familiares de pessoas com TEA precisam lidar com a negação, a culpa, a depressão e o estresse que aquelas questões provocam. Grasu (2018) indicou pesquisas que mostraram o alto nível de estresse e casos de depressão em famílias de pessoas autistas, comparado a outras desordens no desenvolvimento ou pais de crianças com Síndrome de Down, Síndrome do X frágil ou deficiência intelectual severa, sendo que as mães apresentaram os maiores índices de casos de depressão e estresse.

Para enfrentar o desgaste familiar ocasionado pela presença do transtorno, é preciso desenvolver estratégias de enfrentamento (coping). Ooi et al. (2016) estudando pais de autistas notaram que, após aceitarem o diagnóstico de seus filhos, adquiriram conhecimento sobre autismo, muitos tiveram mais pró-atividade nas terapias ou então buscaram um grupo de apoio, apontado como gerador de incentivo e senso de esperança. Ainda, vários pais ganharam esperança ao verem melhoras nos filhos. Outros se apegaram à espiritualidade, enquanto outros passaram a observar o ambiente e a criança, anteciparem respostas e comportamentos da mesma, de modo a preveni-los. Também, foi notado que ter pensamentos positivos 
sobre os filhos melhorou o bem-estar psicológico dos pais e que comportamentos de crianças autistas foram modificados de acordo com o humor de seus pais. Para as autoras, a vida de uma família que tem criança autista é bem diferente de famílias com crianças típicas. No primeiro caso, especialmente, o apoio familiar pode ser uma grande fonte de enfrentamento do TEA, seja o apoio prático ou psicológico (OOI et al, 2016).

Diante disso, Townsend e Puymbroeck (2017) trouxeram estudos que mostraram a importância do lazer para lidar com o estresse gerado em famílias de pessoas com deficiência. Elas apontaram que essas atividades contribuem para o desenvolvimento de consciência dos familiares sobre suas habilidades, de habilidades de negociação, na resolução de problemas e na geração de sistemas de apoio, além de ter havido diversão e fortalecimento de relacionamentos. Após experiência em acampamento com famílias de autistas, ocorreram mudanças profundas no funcionamento familiar: seus membros se mostraram mais interessados em relacionarem-se (coesão), se mostraram mais abertos a fazer atividades juntos (flexibilidade) e melhoraram o diálogo entre os mesmos (comunicação), que passou a ser mais frequente e efetivo. Para as autoras, foi relatada maior satisfação com a vida familiar e com o lazer, que foram expressos pelo compartilhamento das experiências e pelo surgimento de um estado emocional mais positivo na família (TOWSEND; PUYMBROECK, 2017).

Além dos desafios apontados, famílias de pessoas com TEA ainda têm que lidar com o preconceito social sobre o transtorno. Grasu (2018) mencionou que há muita estigmatização social dos autistas, cujos comportamentos são relacionados à indulgência dos pais. Inclusive, muitos pais escolhem matricular seus filhos em turmas de educação especial por medo de que ocorram abusos e por receio de falta de progresso. Entretanto, para Soares (2016), a inclusão escolar da criança autista possibilita o enfrentamento desse preconceito, se realizada de modo adequado. Para a promoção da inclusão de pessoas autistas nas escolas é preciso que a comunidade escolar promova formação sobre TEA, propicie educação especial se necessário, obtenha recursos materiais específicos, envolva o apoio de todos, lide com os sintomas do TEA, administre o tempo escolar propiciando aprendizagens para todos e consiga apoio do Estado, em se tratando de escolas públicas (RORIZ, 2016). O sucesso no desenvolvimento do garoto Pedro, estudado por Cruz (2015), foi relacionado à parceria família e escola, pois além de ter o apoio constante da mãe, inclusive na realização de tarefas escolares em casa, ele também foi auxiliado pela professora de Atendimento Educacional Especializado (AEE) e pela professora da classe regular (CRUZ, 2015).

Também, Mathias e Probst (2016) atribuem à escola a responsabilidade no sucesso da inclusão da criança, considerando o fracasso da aprendizagem discente devido a problemas na intervenção pedagógica. Logo, é preciso que toda comunidade escolar entenda sobre TEA, para que a inclusão de fato ocorra (MATHIAS; PROBST, 2016). Tratando de estudante de faculdade de música, autista e cego, Mousinho, Adour e Gikovate (2016) explicaram que "posturas inflexíveis, restrições pedagógicas e metodológicas e falta de formação e informação não são compatíveis com uma proposta de educação inclusiva, e ainda podem nos privar, e ao mundo, de talentos como ele" (p.204). Nesse caso, os professores do curso focaram em habilidades do estudante e fizeram adaptações necessárias para lidar com o transtorno - uma professora, por exemplo, investiu na construção de uma partitura mental pelo 
estudante, a partir de pistas cognitivas concretas que lhe fornecia e investiu no ensinamento de uso de sinais de acentuação e de sonoridade para interpretação de músicas (MOUSINHO; ADOUR; GIKOVATE, 2016).

Diferente das escolas, o maior desafio de especialistas, segundo os trabalhos analisados, é a dificuldade de dar o diagnóstico. Roriz (2016) mencionou a heterogeneidade, a multifatorialidade e a associação do TEA a comorbidades. "Na maioria das vezes, o prognóstico é difícil de previsão, principalmente que ele considerou doença, nas crianças com menos de três anos, devido ao facto destas não terem desenvolvido todos os sintomas característicos da doença" (RORIZ, 2016, p.56). Assim, o diagnóstico do TEA precisa ser realizado de modo cuidadoso, por meio de acompanhamento clínico e com a utilização de métodos de rastreio e, muitas vezes, com apoio de outros profissionais especialistas.

Diante dos conteúdos apresentados, foi possível observar a importância do conhecimento, da colaboração e do enfrentamento de desafios pela família, pela escola e por especialistas que, em parceria, podem auxiliar no desenvolvimento de pessoas com TEA, ainda que diante da heterogeneidade e da multifatorialidade do transtorno.

\section{Considerações finais}

Essa revisão objetivou analisar estudos que tratavam de potenciais contribuições da família, da escola e de especialistas no desenvolvimento de pessoas com TEA. Foi possível mapear essas colaborações por meio de três categorias: conhecimento, colaboração e desafios.

Sobre a categoria conhecimento, foi observada a imprescindibilidade de se adquirir cognição sobre o TEA por toda a sociedade, visto que ele é compreendido erroneamente pela maioria das pessoas, que 0 restringem a aspectos sintomatológicos e comportamentais disruptivos. É preciso conscientizar acerca do autismo, sendo responsabilidade da família, da escola e de especialistas, os quais, em parceria, devem difundir o entendimento mais integral da pessoa com TEA, suplantando a visão biologizada.

Com relação à categoria colaboração, foi aferida a necessidade de atuação dos três grupos: família, escola e especialistas. A colaboração da primeira consiste em mediar o processo de desenvolvimento do indivíduo com TEA, atendendo sua necessidade de diagnóstico, tratamento de sintomas e inclusão social, além de proceder ao acompanhamento psicológico e a atividades de lazer, evitando o estresse e a depressão.

A escola deve colaborar alicerçando o desenvolvimento cognitivo do indivíduo com TEA, promovendo o desenvolvimento de habilidades sociocognitivas, a partir da estruturação escolar, da adaptação de materiais curriculares, da obtenção de recursos, da gerência de apoio administrativo, de fomento a cursos de capacitação para professores e de ações antibullying. Além disso, é preciso que a escola promova a saúde mental dos adolescentes com TEA por meio do fortalecimento da ideia de pertença desses adolescentes ao grupo social.

Já os especialistas devem colaborar com o tratamento adequado à amenização de sintomas, a partir do processo de diagnóstico e tratamento, além de mediar a relação família, escola e indivíduo, auxiliando os pais no processo de aceitação do 
diagnóstico e orientando e acompanhando os autistas e suas famílias por toda a vida, buscando colaborar com o desenvolvimento da pessoa com TEA.

Para que haja difusão de conhecimento e colaboração entre família, escola e especialistas pelo desenvolvimento da pessoa com TEA é preciso superar os desafios tratados na terceira categoria emergente na pesquisa. A família precisa enfrentar todo o processo de diagnóstico, terapias e convivência com pessoas autistas, o que perpassa em lidar com a prevenção, gerenciamento de comportamentos disruptivos e incômodos, estresse desencadeado pelo preconceito e outros desafios comuns a pessoas com TEA. Ademais, a escola precisa se reestruturar para incluir pessoas com TEA, administrando rotinas, recursos e buscando conhecimento para a comunidade escolar.

Do mesmo modo, o time de especialistas precisa se capacitar e utilizar métodos de rastreio e tratamento adequados aos sintomas, para garantir o diagnóstico e o acompanhamento a pessoas com autismo, devendo, também, compartilhar seus conhecimentos específicos para informar e conscientizar famílias, pessoas autistas e educadores.

Conclui-se, portanto, que é urgente o avanço e a conscientização sobre o TEA, para que sejam promovidas políticas públicas que propiciem apoio aos indivíduos autistas e a suas famílias, a fim de que Ihes sejam garantidos acesso e manutenção a uma rede de auxílio em que família, escola e especialistas possam atuar sistemicamente em prol do desenvolvimento do indivíduo.

\section{Agradecimento}

À Patric Everton da Silva Nascimento pelo auxílio na revisão textual do trabalho.

\section{REFERÊNCIAS}

ALVERSON, Charlotte Y.; LINDSTROM, Lauren E.; HIRANO, Kara A. High School to College: Transition Experiences of Young Adults With Autism. Focus on Autism and Other Developmental Disabilities, v. 34, n. 1, october, p. 52-64, 2015. DOI: https://doi.org/10.1177/1088357615611880

AMERICAN PSYCHIATRIC ASSOCIATION - APA. Manual diagnóstico e estatístico de transtornos mentais: DSM-5.Tradução: Maria Inês Corrêa Nascimento. 5a. ed. Porto Alegre: Artmed, 2014. Disponível em:

http://www.clinicajorgejaber.com.br/2015/estudo_supervisionado/dsm.pdf. Acesso em: 24 jul. 2020.

BARDIN, Laurence. Análise de conteúdo. Lisboa: Edições 70, 1977.

CASANOVA, Maria João Ferreira dos Santos Niz. Um aluno com autismo rumo ao sucesso escolar. 2016. Relatório de Atividade Profissional (Mestrado em Ciências da Educação, especialização em Educação Especial) - Universidade Católica Portuguesa. Faculdade de Filosofia e Ciências Sociais, Braga, Portugal, 2016. Disponível em: https://repositorio.ucp.pt/handle/10400.14/20910. Acesso em: 24 jul. 2020. 
CLARK, Megan; BARBARO, Josephine; DISSANAYAKE, Cheryl. Continuity and Change in Cognition and Autism Severity from Toddlerhood to School Age. Journal Autism Development and Disorders, v. 47, n. 2, p. 328-339, fev. 2017. DOI: https://doi.org/10.1007 / s10803-016-2954-7

CRUZ, Catia Nogueira da. Relações família-escola na educação e no desenvolvimento intelectual de uma criança com diagnóstico de síndrome de espectro autista. 2015. 42 f. Monografia (Especialização em Desenvolvimento Humano, Educação e Inclusão Escolar) - Universidade de Brasília, Universidade Aberta do Brasil, Brasília, 2015. Disponível em:

https://bdm.unb.br/handle/10483/15087. Acesso em: 24 jul. 2020.

DILLON, Gale; UNDERWOOD, Jean; FREEMANTLE, Lauren. Autism and the U.K. secondary school experience. Focus on Autism and Other Developmental Disabilities, p. 1-10, 2014. DOI: https://doi.org/10.1177/1088357614539833

GRASU, Mihaela. Parents against autismo spectrum disorder. Bulletin of Integrative Psychiatry, march, year XXIV, v. 1, n. 76, 2018.

HEYS, Michelle; ALEXANDER, Amy; MEDEIROS, Emilie; TUMBAHANGPHE, Kirti M.; GIBBONS, Felicity; SHRESTHA, Rita; MANANDHAR, Mangala; WICKENDEN, Mary; SHRESTHA, Merina; COSTELLO, Anthony; Manandhar, Dharma; PELLICANO, Elizabeth. Understanding parents' and professionals' knowledge and awareness of autism in Nepal. Autism, v. 21, n. 4, 436-449, maio, 2016.

DOI: https://doi.org/10.1177 / 1362361316646558

LOPES, Claudio Neves. Autismo e Família: O Desenvolvimento da Autonomia de um Adolescente com Síndrome de Asperger e a Relação Familiar. Diálogos e Perspectivas em Educação Especial, v. 5, n.1, p. 53-66, Jan.-Jun., 2018. DOI: https://doi.org/10.36311/2358-8845.2018.v5n1.05.p53

MATIAS, Hélen Bandeira Rosso; PROBST, Melissa. A criança com Transtorno do Espectro Autista, a escola e o professor: algumas reflexões. Profissão DocenteRPD, Uberaba-MG, v.18, n.38, p.158-170, jan./jun. 2018.

DOI: https://doi.org/10.31496/rpd.v18i38.1190

MENDES, Karina Dal Sasso; SILVEIRA, Renata Cristina de Campos Pereira; GALVAO, Cristina Maria. Revisão integrativa: método de pesquisa para a incorporação de evidências na saúde e na enfermagem. Texto \& contexto enfermagem [online]. 2008, vol.17, n.4, pp.758-764. DOI:

http://dx.doi.org/10.1590/S0104-07072008000400018

MOUSINHO,Renata; ADOUR,Andrea; GIKOVATE, Carla. Quem canta, seus males espanta: um ensaio sobre autismo, cegueira, canto, inclusão, superação e sucesso. Revista da Associação Brasileira de Psicopedagogia, v. 33, p. 196-205, 2016. Disponível em: http://www.revistapsicopedagogia.com.br/detalhes/489/quem- 
canta--seus-males-espanta--um-ensaio-sobre-autismo--cegueira--canto--inclusao-superacao-e-sucesso. Acesso em: 24 jul. 2020.

NASCIMENTO, Thamires Thayane Costa do. A inclusão de uma criança com TEA na rede privada de ensino: um estudo de caso sobre a parceria escola \família. 2017. 66 f. Trabalho de Conclusão de Curso (Graduação em Pedagogia) Universidade Federal da Paraíba, João Pessoa/PB, 2017.

OOI, Khim Lynn; ONG, Yin Sin; JACOB, Sabrina Anne; KHAN, Tahir Mehmood. A meta-synthesis on parenting a child with autism. Neuropsychiatric disease and treatment, v. 12, p. 745, 2016. DOI: https://doi.org/10.2147 / NDT.S100634

PERERA, Hemamali; JEEWANDARA, Kamal Chandima; SENEVIRATNE, Sudarshi; GURUGE, Chandima. Outcome of Home-Based Early Intervention for Autism in Sri Lanka: Follow-Up of a Cohort and Comparison with a Nonintervention Group.

BioMed Research International, may, 2016. DOI:

https://doi.org/10.1155/2016/3284087

RAVET, Jackie. But how do I teach them?': Autism \& Initial Teacher Education (ITE). International Journal of Inclusive Education, 2017. DOI:

https://doi.org/10.1080/13603116.2017.1412505

\section{RIBEIRO, Tatiana de Farias Ramos. Um olhar sobre o autismo: inclusão} escolar, conceitos e discussões. 2018. $33 \mathrm{f}$. Trabalho de conclusão de curso de graduação em Pedagogia - Universidade Estadual da Paraíba, Campina Grande, 2018. Disponível em:

http://dspace.bc.uepb.edu.br/jspui/handle/123456789/17585. Acesso em: 24 jul. 2020.

RORIZ, Diana Catarina Amorim. Autismo: o doente a família e a sociedade. 2016. 111f. Dissertação (Mestrado Integrado em Medicina), Faculdade de Medicina, Universidade de Coimbra- Portugal, 2016. Disponível em: https://estudogeral.sib.uc.pt/handle/10316/33722. Acesso em: 24 jul. 2020.

SANTOS, Lucas Luiz Nogueira; LEÃO, Marcelo Franco; AGAPITO, Francica Melo. Atendimento a estudantes com autismo em um município de Mato Grosso: Com a palavra os profissionais da educação. Pesquisa em foco (UEMA), v. 23, p. 118141, 2018. DOI: https://doi.org/10.18817/pef.v23i1.1656

SHOCHET, Ian; SAGGERS, Beth; CARRINGTON, Suzanne; ORR, Jayne; WURFL, Astrid; DUNCAN, Bonnie; SMITH, Coral. The Cooperative Research Centre for Living with Autism (Autism CRC) Conceptual Model to Promote Mental Health for Adolescents with ASD. Clinical Child and Family Psychology Review, v. 19, n. 2, p.94-116, 2016. DOI: https://doi.org/10.1007 / s10567-016-0203-4

SILVA, Ana Beatriz Barbosa; GAIATO, Mayra Bonifácio; REVELES, Leandro Tadeu. Mundo singular: Entenda o Autismo. Fontanar, 2012. 
SOARES, Patrícia da Rosa. O sujeito autista e a inclusão escolar: a importância do enlace entre família, escola e psicólogo. 2016. 35 f. Trabalho de Conclusão de Curso (Graduação em Psicologia) - Universidade Regional do Noroeste do Estado do Rio Grande do Sul (UNIJUÍ), Ijuí, 2016. Disponível em:

http://bibliodigital.unijui.edu.br:8080/xmlui/handle/123456789/4296. Acesso em: 24 jul. 2020.

SOUZA, Marcela Tavares de; SILVA, Michelly Dias da; CARVALHO, Rachel de. Revisão integrativa: o que é e como fazer. Einstein, v. 8, p. 102-106, 2010. DOI: https://doi.org/10.1590/s1679-45082010rw1134

TOWNSEND, Jasmine; PUYMBROECK, Marieke Van. Parental Perceptions of Changes in Family Well-Being Following Participation in a Camp Experiences of Families With a Child With ASD. Therapeutic Recreation Journal, v. 51, n. 2, p. 143-163, 2017. DOI: https://doi.org/10.18666/TRJ-2017-V51-I2-8359

Recebido em: 22 de setembro de 2020. Aceito em: 11 de novembro de 2020. Publicado em: 05 de janeiro de 2021. 